The current approach unfortunately contains none of these elements, but the resources to do so are available. We are experiencing a revolution in our ability to predict disease, track its progress, and intervene coherently. These new capacities, stemming in part from genomics, systems biology, and advanced medical technologies, can improve care by making it personalized, predictive, preventive, and costeffective. Capabilities are being improved to define each individual's risk for developing chronic diseases as well as to detect the onset and provide the best personalized treatment when needed. We already have the know-how to create strategic solutions to improving health and minimizing disease (1). Such approaches, called prospective care, personalized medicine, integrative care, or patient-centered care, provide individuals with an assessment of their health risks and the education and support needed to create their strategic health plan. This is facilitated by an ongoing relationship with a health system that provides a primary point of contact; i.e., primary care physician and/or health care coach and coordinated care over time. These capabilities can be far more cost-effective, as they heighten the involvement and compliance of the individual and organize care over time. In contrast, the system today focuses on poorly coordinated and expensive treatment of episodes of late-stage chronic disease, many of which are preventable.

As a physician with 40 years' experience in virtually all aspects of medicine, I believe that our profession has an obligation to provide a strong voice in the health care debate. My belief is that the needed outcomes for a rational approach to care include:

Accessibility. Health is among our greatest resources and a basic value. Access and insurance to support health and minimize and treat disease should be available for all Americans.

Education. Educating the public about the importance of health and one's ability to influence it should be a major societal focus. Why not advocate a broad "going healthy" movement similar to the "going green" initiative to focus our nation on improving health as a national goal and a shared responsibility? Businesses, schools, communities, consumer groups, social and religious organizations, public health groups, and the government could develop collaborations focusing on improving our nation's health.

Approach to care. Strategic health planning based on a patient's risks for preventable diseases along with appropriate tracking and early personalized intervention are crucial. Chronic diseases develop over time, so there are abundant opportunities for personalization, prevention, and minimization of disease progression. The basic medical work-up should move from its current "find it and fix it" mind-set to one that is also proactive and preventive.

Delivery systems. Health providers should be organized to give the patient ongoing and coherent care at the level needed, from health promotion to treatment of disease events.

Biomedical research and continuous improvement. Prediction, prevention, and personalization are hallmarks of rational health care. These capabilities require constant advancement, and thus support is needed for robust biomedical research and translation. Regulatory processes should be created to allow rapid adoption of validated discoveries.

Reimbursement. Strategies to pay for prevention, continuity of care, and better outcomes are essential. Our current system rewards interventions for disease events and thus encourages the care we have today. Reimbursement for prevention, early intervention, and effective long-term management is missing. Of all the impediments to reform, changes in reimbursement may be the most difficult to overcome, as there is so much money at stake. Nonetheless, reimbursing pro- viders for better clinical outcomes will have the greatest leverage in fostering better models of care.

Creating and validating better care models. While the concepts of personalized, prospective care are sound and indications of effectiveness apparent, we must develop more working models and learn from those that work the best. To stimulate competition and ingenuity, demonstration projects funded by the government and private insurers should be established. Outcomes could be monitored and overseen by credible, nonbiased entities such as the Institute of Medicine.

I believe that as a nation, we must affirm our commitment to a reasonable and practical level of health care as an individual's right. Health care must be directed toward enhancing patient involvement to improve their health along with systems to support this. Personalized, predictive, preventive, and coordinated strategies can replace the current approach, which wastefully focuses on disease events. I consider our health care system to be the best in the world in dealing with disease events, yet sorely inadequate in preventing and minimizing disease - we can and we must do better. Resolving this dilemma is at the crux of meaningful health care reform, and, as physicians, we must provide a voice of reason in this debate.

\section{Ralph Snyderman}

Duke University Medical Center, Durham, North Carolina, USA. E-mail: ralph. snyderman@duke.edu.

Conflict of interest: Ralph Snyderman is chairman of Proventys Inc.

J. Clin. Invest. 119:2855-2856 (2009). doi:10.1172/JCI41034.

1. Snyderman, R. 2004. The AAP and the transformation of medicine. J. Clin. Invest. 114:1169-1173.

\title{
Health care reform - need for less emotion and more science
}

he United States is currently engaged in a very important debate on the issue of health care reform. There is much rhetoric about the dangers of reform, the negative role of government versus the private sector in administering health care, the risk of losing personal control over important health care choices, the merits and weaknesses of a single-payer system, the value of 
free markets versus regulated markets, and the likelihood of higher costs no matter what we do. As physicians, we all want the quality of medical care in the United States to be as high as possible, and with a reasonable level of medical care available for everyone. As medical scientists, we should also insist that as we make this decision, we look at the options using as scientific an approach as possible.

\section{The physician's voice}

Compared with most European countries, Canada, and Japan, life expectancy in the US is shorter, and rates of infant mortality, maternal mortality, and adult mortality are higher (3). This occurs despite the fact that the US spends more of its gross domestic product per capita on health care than any country in the world.

The US is among the most fortunate countries in the world in terms of health care resources. We have one of the strongest academic medical communities in the world. There are rigorous standards of medical education and training, resulting in excellent physicians providing highquality medical care. US hospitals lead the world in application of the newest and most sophisticated approaches. The US is also the leader in the development of pharmaceuticals, biologicals, and new technologies for medical care, which are often available in the US earlier and to a greater extent than in other countries.

Despite this, the US does not lead the world in major metrics of health care effectiveness or access. There is a large segment of the population that is uninsured, even compared to countries where private insurance is common (less than $0.2 \%$ of legal Swiss or German residents are uninsured compared with nearly $18 \%$ of Americans) (1, 2). Furthermore, compared with most European countries, Canada, and Japan, life expectancy in the US is shorter, and rates of infant mortality, maternal mortality, and adult mortality are higher (3). This occurs despite the fact that the US spends more of its gross domestic product per capita on health care than any country in the world.
So why do these discrepancies exist, and what should be done to rectify them? The reasons are certainly multiple. One factor often cited for the high cost of medicine is the fact that the average physician's salary is higher in the US by $30 \%-50 \%$ than in most European countries $(1,2)$. However, average salaries in almost all professions are higher in the US, and the number of physicians per 1,000 residents is proportionally lower in the US than in most European countries (3), so the total cost of physician services is actually similar. This of course raises the question as to whether the US physician works proportionally harder to cover her/his patient load than the European physician, or whether the patient has less physician time in the US than in Europe, and whether this has an impact on ultimate outcome. The issues of higher pharmaceutical costs, higher procedure utilization and costs, higher hospital costs, and the costs of practicing defensive medicine due to the risk of malpractice suits in the US compared to other countries likewise need to be addressed with data. In each case, we need to consider not only the financial impact of our approach versus other approaches, but also which activities provide a meaningful benefit to the patient and which do not.

One area where Americans pay more than residents of any other country is the high administrative overhead created by the current health care system. Indeed, the US leads all industrialized countries in the share of national health care expenditures devoted to administration, and there is no evidence that this extra administration adds any value to the system. In fact, most physicians and patients would agree that dealing with insurance companies over issues of eligibility for care is made intentionally difficult and time consuming as a way to discourage, not improve, care. This is not a trivial issue. In the US, the administrative share is more than three times that of Japan or Canada. An analysis published in the New England Journal of Medicine in 2003 (4) using data from 1999 estimated that Americans spent \$1,059 per capita on the administrative costs of health care (insurers' costs, the costs borne by employers, health care providers, and governments) compared with only \$307 in purchasing power parity dollars spent in Canada. When calculated in today's terms, the total excess spending for health care administration in the US is between $\$ 150$ billion and $\$ 220$ billion annually - far more than enough to finance universal health insurance or many other benefits - and this doesn't even include the value of the patient's time trying to get coverage or claim reimbursement. Unfortunately, with the strong lobbying system in the US, even obvious issues that could be addressed, such as this one, are often obscured by all of the "noise" created by special interest groups.

I believe that as physician-scientists, rather than each of us taking a stance on health care reform based on our feelings, we should push the principles of evidencebased medicine to gather appropriate data by which to make these decisions. Thus, we need to perform studies, not just comparing treatment A to treatment B, but comparing health care system $C$ to health care system D. These comparisons need to be designed as real experiments and include the full range of options from individual choice to a single-payer, government-run plan. This would allow us to answer questions as to whether the metrics of health outcome and performance are better or worse in one system of care versus another. Without such data, mandating any single national system of care is risky at best. I have little doubt that by allowing different states or other large cross-sectional population blocks to try different experiments in health care delivery over the next decade, we could define a health care system for the US that will be not only lower in cost, but much more effective and inclusive than the current system.

\section{Ronald Kahn}

Joslin Diabetes Center and Harvard Medical School, Boston, Massachusetts, USA. E-mail: c.ronald.kahn@joslin.harvard.edu.

J. Clin. Invest. 119:2856-2857 (2009). doi:10.1172/JCI41036.

\footnotetext{
1. Shi, L., and Singh, D.A. 2007. Delivering health care in America: a systems approach. Jones and Bartlett Publishers. Sudbury, Massachusetts, USA. 650 pp.

2. McPake, B., and Normand, C. 2008. Health economics: an international perspective. 2nd edition. Routledge, New York, USA. 292 pp.

3. WHO Statistical Information System. http://apps. who.int/whosis/data/Search.jsp.

4. Woolhandler, S., Campbell, T., and Himmelstein, D.U. 2003. Costs of health care administration in the United States and Canada. N. Engl. J. Med. 349:768-775.
} 